\title{
UMA BREVE ANÁLISE DO CICLO DE PREMIAC̣ÃO 1999
}

\author{
Patricia Laczynski, Ricardo Ernesto Vasquez Beltrão e Marta Ferreira Santos Farah
} (Membros da Equipe de Coordenação do Programa GESTÃO PÚBLICA E CIDADANIA)

O Programa GESTÃo PÚBLICA E CIDADANIA completou em 1999 quatro anos, totalizando neste período 2.445 programas, projetos ou atividades de instituições públicas governamentais dos níveis estadual e municipal e das organizações próprias dos povos indígenas. Só em 1999 foram 888 iniciativas inscritas, o que representa um aumento de $40,7 \%$ em relação ao número de inscrições do ano anterior.

TABELA 1 - DISTRIBUIÇÃO DOS PROGRAMAS, PROJETOS E ATIVIDADES INSCRITOS, POR NIVEL DE GOVERNO - CICLOS DE PREMIAÇÃO 1996, 1997, 1998 E 1999

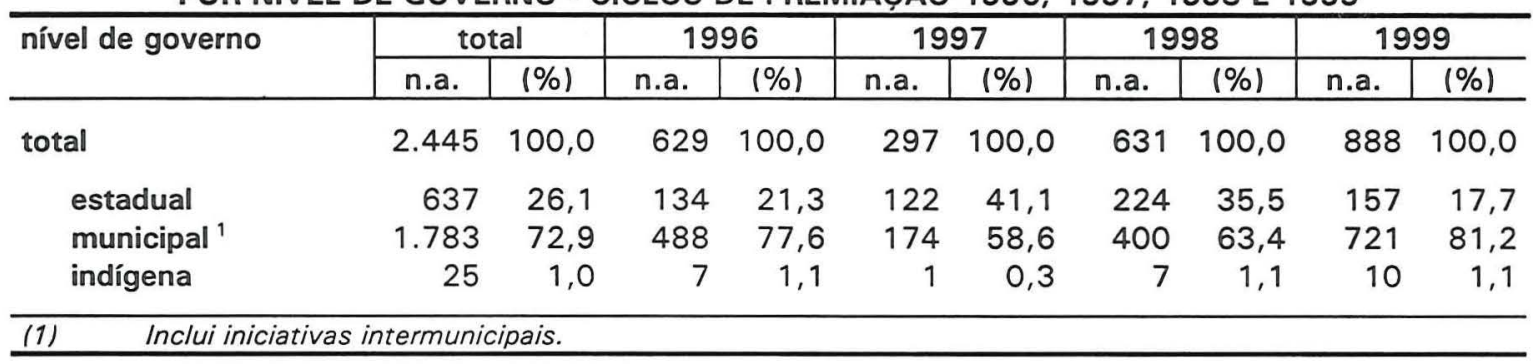

A evolução das participações nos quatro anos de funcionamento do Programa GESTÃO PÚBLICA E CIDADANIA pode ser vista nas tabela 1 e 2 . São as iniciativas municipais que compõem a maioria das inscrições. Em 1999 esse número foi de 721 inscrições, $81,2 \%$ do total. Se compararmos com o ano de 1998 , percebemos que as inscrições de iniciativas estaduais diminuíram 29,9\% e as municipais aumentaram $80,3 \%$. Isso reforça a hipótese, trabalhada nos anos anteriores, de que no primeiro ano de governo o número de inscrições tende a diminuir. Assim como as inscrições de iniciativas estaduais diminuíram em 1999 - primeiro ano de gestão dos governos estaduais -, as municipais diminuíram em 1997 (64,3\%) - primeiro ano de administração dos governos municipais.

As inscrições de iniciativas próprias dos povos indígenas mantiveram a mesma porcentagem entre 1998 e 1999, o que significou um aumento total de sete para dez 
UMA BREVE ANÁLISE DO CICLO DE PREMIAÇÃO 1999

inscrições. Desde 1997, quando as inscrições diminuíram de sete para uma, a coordenação do Programa tem buscado divulgar entre os povos indígenas o Ciclo de Premiação. Para tanto elaborou, em parceria com o Núcleo de Cultura Indígena, um folder com linguagem específica e um calendário "aberto" (quando as suas inscrições não cumprem o prazo determinado pelo Programa GESTÃO PÚBLICA E CIDADANIA, elas são validadas automaticamente para o Ciclo de Premiação do ano seguinte), viabilizando a maior participação destas iniciativas.

TABELA 2 - VARIAC̣ÃO PERCENTUAL DO NÚMERO DE INSCRIÇÕES POR NÍVEL DE GOVERNO CICLOS DE PREMIAC̣ÃO 1996, 1997, 1998 E 1999

\begin{tabular}{l|r|r|r}
\hline nível de governo & de 1996 para 1997 & de 1997 para 1998 & de 1998 para 1999 \\
\hline total & $-52,8$ & $+112,5$ & $+40,7$ \\
\multicolumn{1}{c|}{ estadual } & $-9,0$ & $+83,6$ & $-29,9$ \\
municipal $^{1}$ & $-64,3$ & $+129,9$ & $+80,3$ \\
\hline (1) Inc/ui iniciativas intermunicipais. & & & \\
\hline
\end{tabular}

Foram 21 os governos estaduais que inscreveram programas, projetos ou atividades no Ciclo de Premiação 1998. Em 1999, este número caiu para 19. Mesmo assim, cinco governos estaduais - AM, PA, RN, RO e SE - que não haviam inscrito nenhuma iniciativa estadual no ano anterior, participaram em 1999.

Em relação aos governos municipais, eles totalizaram 228 com inscrições municipais e intermunicipais, o que representou um aumento de 62,9\% em relação a 1998 (140 governos municipais). Do total dos governos municipais que participaram em 1999, 52,9\% ainda não haviam participado de nenhum Ciclo de Premiação nos três anos anteriores.

Este quadro reflete o esforço por parte da coordenação do Programa em difundir os Ciclos de Premiação junto aos governos subnacionais. Estimular a participação destes no Programa GESTÃO PÚBLICA E CIDADANIA, focalizando o que está indo bem na administração pública, descobrindo as inovações, e aumentando o estoque de conhecimento sobre experiências alternativas em gestão pública, para depois disseminá-las, é o grande objetivo do Programa.

Para além do esforço de divulgação, essa participação significativa pode estar ancorada na atuação cada vez maior dos níveis subnacionais de governos na gestão e oferta de serviços públicos, fortemente impulsionada com a Constituição de 1988. 
A maior parte das inscrições no Ciclo de Premiação 1999 é da região Sudeste, totalizando $51,0 \%$ do total. Em seguida, vem a região Sul, com 29,6\%. Essas duas regiões têm concentrado a maioria das iniciativas inscritas nesses quatro anos de Programa, correspondendo a $78,0 \%$ de todas as inscrições. O Norte é a região com menos inscrições, apesar do aumento de 26 para 38 entre 1998 e 1999 (vide tabela 3).

TABELA 3 - DISTRIBUIC̣ÃO DOS PROGRAMAS, PROJETOS E ATIVIDADES INSCRITOS, POR REGIÃO GEOGRÁFICA - CICLOS DE PREMIAÇÃO 1996, 1997, 1998 E 1999

\begin{tabular}{|c|c|c|c|c|c|c|c|c|c|c|}
\hline \multirow[t]{2}{*}{ região } & \multicolumn{2}{|c|}{ total } & \multicolumn{2}{|c|}{1996} & \multicolumn{2}{|c|}{1997} & \multicolumn{2}{|c|}{1998} & \multicolumn{2}{|c|}{1999} \\
\hline & n.a. & $(\%)$ & n.a. & (\%) & n.a. & $(\%)$ & n.a. & $(\%)$ & n.a. & $(\%)$ \\
\hline Total & 2.448 & 100,0 & 629 & 100,0 & 297 & 100,0 & 631 & 100,0 & 888 & 100,0 \\
\hline Norte & 89 & 3,6 & 17 & 2,7 & 8 & 2,7 & 26 & 4,1 & 38 & 4,3 \\
\hline Nordeste & 309 & 12,6 & 70 & 11,1 & 61 & 20,5 & 60 & 9,5 & 116 & 13,1 \\
\hline Sudeste & 1.282 & 52,4 & 384 & 61,1 & 120 & 40,4 & 324 & 51,4 & 453 & 51,0 \\
\hline Sul & 627 & 25,6 & 134 & 21,3 & 62 & 20,9 & 168 & 26,6 & 263 & 29,6 \\
\hline Centro-Oeste & 141 & 5,8 & 24 & 3,8 & 46 & 15,5 & 53 & 8,4 & 18 & 2,0 \\
\hline
\end{tabular}

A participação da região Nordeste quase dobrou em relação ao Ciclo de 1998 , com aumento de 60 para 116 inscrições. O Estado de Alagoas é o único que permanece, desde 1996, sem nenhuma iniciativa enviada pelo nível estadual de governo, o que talvez seja reflexo da grave crise fiscal enfrentada por este Estado há muitos anos. De qualquer forma, isto sugere que o programa GESTÃO PÚBLICA E CIDADANIA deve investir ainda mais na divulgação neste Estado, atraindo-o para participar dos próximos Ciclos de Premiação.

Se as regiões Sudeste e Sul foram as que mais apresentaram iniciativas inscritas no Ciclo de Premiação 1999, nada mais lógico do que municípios destas regiões se destacarem quanto ao número de iniciativas municipais. São eles: Porto Alegre/RS (44), Santo André/SP (24), Betim/MG (20), Chapecó/SC (16), Presidente Prudente/SP (14), Rio de Janeiro/RJ (14), Juiz de Fora/MG (14), Franca/SP (13), Belo Horizonte/MG (12), Jaraguá do Sul/SC (12), Blumenau/SC (11), Joinville/SC (11), Cantagalo/RJ (10), Lages/SC (10) e João Monlevade/MG (10). Mas municípios de outras regiões também apresentaram números elevados de inscrições, com destaque para Camaçari/BA (15), Camaragibe/PE (12) e Teresina/PI (10). 
UMA BREVE ANÁLISE DO CICLO DE PREMIAC̣ÃO 1999

\begin{tabular}{|c|c|c|c|c|c|c|}
\hline \multicolumn{7}{|c|}{$\begin{array}{l}\text { TABELA } 4 \text { - LOCALIDADES QUE ENVIARAM APENAS UMA OU DUAS INSCRIÇÕES, } \\
\text { POR NÍVEL DE GOVERNO - CICLOS DE PREMIAC̣ÃO } 1998 \text { E } 1999\end{array}$} \\
\hline \multirow[t]{2}{*}{ número de inscrições } & \multicolumn{3}{|c|}{1998} & \multicolumn{3}{|c|}{1999} \\
\hline & total & estadual & Municipal & total & estadual & municipal \\
\hline uma inscrição & 83 & 5 & 78 & 124 & 5 & 119 \\
\hline duas inscrições & 22 & 6 & 16 & 46 & 6 & 40 \\
\hline total & 105 & 11 & 94 & 170 & 11 & 159 \\
\hline
\end{tabular}

Por outro lado, 159 governos municipais inscreveram apenas uma ou duas iniciativas, o que representa $69,1 \%$ de aumento em relação a 1998, quando esse número era de 94 (vide tabela 4). Já o crescimento do número de governos municipais que inscreveram três ou mais inscrições neste mesmo período foi de 50,0\% (de 46 para 69 governos municipais). Este quadro sugere algumas hipóteses que podem ser desenvolvidas em futuros estudos, como por exemplo, um maior esforço de governos municipais em inscreverem políticas e programas mais abrangentes que contemplem diversas atividades, ou ainda, uma seletividade mais rígida por parte dos governos locais ao inscreverem seus programas.

TABELA 5 - DISTRIBUIÇÃO DAS LOCALIDADES ONDE SÃO DESENVOLVIDAS AS INICIATIVAS INSCRITAS, POR INTERVALOS DE NÚMERO DA HABITANTES, SEGUNDO NIVEIS DE GOVERNO - CICLOS DE PREMIACCÃO 1998 E 1999

\begin{tabular}{|c|c|c|c|c|c|c|c|c|c|c|c|c|}
\hline \multirow{3}{*}{$\begin{array}{l}\text { número de } \\
\text { habitantes } \\
\text { (em milhares) }\end{array}$} & \multicolumn{6}{|c|}{1998} & \multicolumn{6}{|c|}{1999} \\
\hline & \multicolumn{2}{|c|}{ total } & \multicolumn{2}{|c|}{ estaduais (1) } & \multicolumn{2}{|c|}{ municipais ${ }^{(2)}$} & \multicolumn{2}{|c|}{ total } & \multicolumn{2}{|c|}{ estaduais } & \multicolumn{2}{|c|}{ municipais ${ }^{(2)}$} \\
\hline & n.a. & (\%) & n.a. & (\%) & n.a. & (\%) & n.a. & $(\%)$ & n.a. & (\%) & n.a. & $(\%)$ \\
\hline TOTAL & 208 & 100,0 & 62 & 29,8 & 146 & 70,2 & 284 & 100,0 & 56 & 19,7 & 228 & 80,3 \\
\hline até 20 & 43 & 20,7 & 8 & 3,8 & 35 & 16,8 & 76 & 26,8 & 7 & 2,5 & 69 & 24,3 \\
\hline+ de 20 até 50 & 27 & 13,0 & 4 & 1,9 & 23 & 11,1 & 50 & 17,6 & 4 & 1,4 & 46 & 16,2 \\
\hline + de 50 até 100 & 35 & 16,8 & 7 & 3,4 & 28 & 13,5 & 40 & 14,1 & 5 & 1,8 & 35 & 12,3 \\
\hline + de 100 até 200 & 25 & 12,0 & 7 & 3,4 & 18 & 8,7 & 33 & 11,6 & 5 & 1,8 & 28 & 9,9 \\
\hline+ de 200 até 500 & 30 & 14,4 & 7 & 3,4 & 23 & 11,1 & 40 & 14,1 & 9 & 3,2 & 31 & 10,9 \\
\hline+ de 500 até 1.000 & 11 & 5,3 & 5 & 2,4 & 6 & 2,9 & 9 & 3,2 & 2 & 0,7 & 7 & 2,5 \\
\hline+ de 1.000 até 5.000 & 20 & 9,6 & 12 & 5,8 & 8 & 3,8 & 19 & 6,7 & 13 & 4,6 & 6 & 2,1 \\
\hline+ de 5.000 até 10.000 & 9 & 4,3 & 7 & 3,4 & 2 & 1,0 & 8 & 2,8 & 6 & 2,1 & 2 & 0,7 \\
\hline+ de 10.000 & 4 & 1,9 & 4 & 1,9 & -- & -- & 5 & 1,8 & 5 & 1,8 & -- & -- \\
\hline sem informação & 4 & 1,9 & 1 & 0,5 & 3 & 1,4 & 4 & 1,4 & -- & -- & 4 & 1,4 \\
\hline $\begin{array}{l}\text { (1) Inclui iniciativas } \\
\text { municipal ou regional foi } \\
\text { projeto ou atividade. } \\
\text { (2) Inclui iniciativas }\end{array}$ & & al & rqar & & rón & & & (1) & & & & nce \\
\hline
\end{tabular}

No total, em 1999, foram 284 localidades que inscreveram programas, projetos ou atividades, 36,5\% a mais que em 1998 (208 localidades), conforme a tabela 5. Para este cálculo, o número de habitantes, no caso das iniciativas estaduais, é considerado do ponto 
UMA BREVE ANÁLISE DO CICLO DE PREMIAÇÃO 1999

de vista da abrangência geográfica das experiências, para diferenciar aquelas iniciativas que são desenvolvidas por órgão público estadual em apenas uma cidade ou região específica das que dizem respeito ao Estado por inteiro. Por exemplo, quando a iniciativa estadual for de São Paulo e sua abrangência geográfica for apenas o município de Bauru, consideramos a população desta localidade, diferentemente das iniciativas estaduais de São Paulo, cuja abrangência é o Estado por inteiro.

Seguindo uma tendência já evidenciada em 1998, o maior crescimento do número de inscrições se verificou entre as cidades com até 50 mil habitantes. 0 número de inscrições das localidades com até 20 mil habitantes apresentou um aumento de 76,7\%, pois de 1998 para 1999 o número de inscrições saltou de 43 para 76. Das localidades de 20 mil a 50 mil habitantes, este crescimento foi de $85,2 \%$ (27 para 50 inscrições). Isso sugere a hipótese de um empenho pela busca da inovação, cada vez mais acentuado.

TABELA 6 - PROGRAMAS, PROJETOS E ATIVIDADES INSCRITOS POR NÍVEL DE GOVERNO SEGUNDO O PERÍODO DE IMPLEMENTACCÃO - CICLOS DE PREMIAÇÃO 1997, 1998 E 1999 (*)

\begin{tabular}{|c|c|c|c|c|c|c|c|c|c|c|c|c|}
\hline \multirow{3}{*}{$\begin{array}{l}\text { GESTÃO GOVERNAMENTAL } \\
\text { RESPONSÁVEL PELA INSCRIÇÃO }\end{array}$} & \multicolumn{12}{|c|}{ ANO DO CICLO DE PREMIAÇÃO E NÍVEL DE GOVERNO } \\
\hline & \multicolumn{3}{|c|}{ TOTAL } & \multicolumn{3}{|c|}{1997} & \multicolumn{3}{|c|}{1998} & \multicolumn{3}{|c|}{1999} \\
\hline & total & $\begin{array}{l}\text { esta- } \\
\text { dual }\end{array}$ & $\begin{array}{c}\text { muni- } \\
\text { cipal }\end{array}$ & total & $\begin{array}{l}\text { esta- } \\
\text { dual }\end{array}$ & $\begin{array}{c}\text { muni- } \\
\text { cipall }\end{array}$ & total & $\begin{array}{l}\text { esta- } \\
\text { dual }\end{array}$ & \begin{tabular}{|c|} 
muni- \\
cipal
\end{tabular} & total & $\begin{array}{l}\text { esta- } \\
\text { dual }\end{array}$ & $\begin{array}{l}\text { muni- } \\
\text { cipal }\end{array}$ \\
\hline TOTAL & 98 & 506 & 1292 & 296 & 125 & 171 & 624 & 224 & 400 & 878 & 157 & 721 \\
\hline \multirow{3}{*}{$\begin{array}{l}\text { gestão atual (1) } \\
\text { gestão antecessora (3) } \\
\text { antes da gestão antecessora (4) }\end{array}$} & 946 & 245 & 701 & 85 & 85 & $\ldots(2)$ & 343 & 160 & 183 & 518 & .- (2) & 518 \\
\hline & 606 & 171 & 435 & 159 & 30 & 129 & 199 & 37 & 162 & 248 & 104 & 144 \\
\hline & 246 & 90 & 156 & 52 & 10 & 42 & 82 & 27 & 55 & 112 & 53 & 59 \\
\hline DISTRIBUIÇÃO PERCENTUAL & 100,0 & 100,0 & 100,0 & 100,0 & 100,0 & 100,0 & 100,0 & 100,0 & 100,0 & 100,0 & 100,0 & 100,0 \\
\hline gestão atual (1) & 52,6 & 48,4 & 54,3 & 28,7 & 68,0 & $--(2)$ & 55.0 & 71,4 & 45,8 & 59.0 & $--(2)$ & 71,8 \\
\hline gestão antecessora (3) & 33,7 & 33,8 & 33,7 & 53,7 & 24,0 & 75,4 & 31,9 & 16,5 & 40,5 & 28,2 & 66,2 & 20.0 \\
\hline antes da gestão antecessora (4) & 13,7 & 17,8 & 12,1 & 17,6 & 8,0 & 24,6 & 13,1 & 12,1 & 13,7 & 12,8 & 33,8 & 8,2 \\
\hline \multicolumn{13}{|c|}{$\begin{array}{l}\text { Não inclui iniciativas protagonizadas por organizaçóes próprias dos povos indigenas. } \\
\text { Para os governos estaduais, quadrienio } 1995-1998 \text { (até o ano de } 1998 \text { ) e } 1999-2002 \text { (para o ano de } 1999 \text { ). para os }\end{array}$} \\
\hline $\begin{array}{l}\text { Para os governos estaduais, } \\
\text { municipais, } 1997-2000 .\end{array}$ & quadriên & io 1995 & 5-1998 & laté o a & no de & 1998), e & e 1999 & 2002 & para o a & ano de & 1999): & para os \\
\hline Năo se aplica porque o Progra & pa exi & E $\mathrm{dr}$ & $e f$ & ivo func & ionamer & & & & & & & \\
\hline $\begin{array}{l}\text { Para os governos estaduais, } \\
\text { municipais, 1993-1996. }\end{array}$ & uadrie & io 1991 & $1-1994$ & laté o a & ano de & 1998), & 199 & 9981 & & & 1999); & \\
\hline $\begin{array}{l}\text { Para os governos estaduais, } \\
1992 .\end{array}$ & té 1990 & $O$ laté & ano de & (1998), & $e$ até & $1994 / p$ & para 0 & & 1999); $p$ & para os & munic & \\
\hline
\end{tabular}


UMA BREVE ANÁLISE DO CICLO DE PREMIAÇÃO 1999

Dos programas, projetos e atividades inscritos no Ciclo de Premiação 1999, 189 deles, ou seja 21,3\%, tiveram ó ano de 1998 como data de início. Em 1998 foram 45,8\% as experiências inscritas com um ano de existência, o que pode significar uma mudança de perfil. Segundo a tabela 6 , contudo, podemos perceber que a maioria dos programas, projetos e atividades foi implementado durante a própria gestão dos governos responsáveis pelas inscrições. Isso não se verificou apenas em 1997 (para os governos municipais) e em 1999 (para os estaduais), o que se explica pela exigência de um ano de implementação para inscrição no Programa, inviabilizando que governos recém-empossados enviem material relativo a iniciativas por sua própria iniciativa.

Ao longo dos três últimos Ciclos de Premiação (não foi possivel levantar os dados para 1996), 52,6\% das iniciativas foram implementadas durante a própria gestão responsável pela inscrição. Em 1999, dos programas, projetos e atividades municipais inscritos, $71,8 \%$ foram implantados na atual gestão. É preciso cuidado ao fazer inferências sobre o padrão de continuidade de políticas públicas a partir destes resultados. Podemos, no entanto, levantar a hipótese de que os governos se sentem mais estimulados a inscreverem programas implementados por sua própria iniciativa, particularmente em ano pré-eleitoral, quando muitos programas estão consolidados.

Mas é importante ressaltar, de qualquer forma, que estudo realizado em 1997, após mudança de governos municipais, por Spink, Clemente e Keppke (1999), junto a 274 programas, projetos e atividades inscritos no Ciclo de Premiação 1996, apurou que 88,0\% deles haviam continuado no governo seguinte, mesmo com a mudança de partido político no poder em metade dos casos.

TABELA 7 - DISTRIBUIÇÃO DAS INICIATIVAS INSCRITAS, PRÉ-FINALISTAS E FINALISTAS SEGUNDO AS GRANDES ÁREAS DE AC̣ÃO GOVERNAMENTAL - CICLO DE PREMIAC̣ÃO 1999

\begin{tabular}{l|rr|r|r|r|r|r|r}
\hline \multirow{2}{*}{ ÁREAS DE GOVERNO } & \multicolumn{2}{|c|}{ inscritos } & \multicolumn{2}{c|}{ semifinalistas } & \multicolumn{2}{c|}{ pré-finalistas } & \multicolumn{2}{c}{ finalistas } \\
\cline { 2 - 9 } n.a. & (em \%) & n.a. & (em \%) & n.a. & (em \%) & n.a. & (em \%) \\
\hline \multirow{2}{*}{ Total } & 888 & 100,0 & 100 & 100,0 & 30 & 100,0 & 20 & 100,0 \\
serviços públicos & 401 & 45,1 & 39 & 39,0 & 9 & 30,0 & 6 & 30,0 \\
cidadania e direitos humanos & 228 & 25,7 & 19 & 19,0 & 6 & 20,0 & 5 & 25,0 \\
administração e governo & 90 & 10,1 & 9 & 9,0 & 4 & 13,3 & 4 & 20,0 \\
desenvolvimento econômico e social & 85 & 9,6 & 27 & 27,0 & 9 & 30,0 & 3 & 15,0 \\
infra-estrutura e meio ambiente & 77 & 8,7 & 6 & 6,0 & 2 & 6,7 & 2 & 10,0 \\
judiciário & 4 & 0,5 & -- & -- & -- & -- & -- & -- \\
legislativo & 3 & 0,3 & -- & -- & -- & -- & -- & -- \\
\hline
\end{tabular}


Em 1999, os programas da área "Desenvolvimento Econômico e Social" representaram $9,6 \%$ do total de programas inscritos; os programas semifinalistas desta área representaram $27 \%$ dos programas semifinalistas; enquanto os programas pré-finalistas e finalistas desta mesma área representaram 30,0\% e 15,0\% do total, respectivamente (vide tabela 7). Isto demonstra ser esta uma área em que estão emergindo programas de boa qualidade.

É interessante observar como a zona rural tem atraído a atenção dos governos locais. Dos 30 programas pré-finalistas em 1999, 13 têm atuação na zona rural (43,3\%), sendo que nove foram classificados entre os 20 finalistas $(45,0 \%)$. Nove programas pré-finalistas têm como área de atuação "Desenvolvimento Econômico e Social", sendo que sete deles têm atuação na zona rural $(77,8 \%)$. Isto sugere que a zona rural esteja sendo repensada pelos governos locais como espaço de desenvolvimento sustentável.

As subáreas com mais inscrições são "Criança e Adolescente", "Educação", "Saúde", "Assistência Social", e "Formação de Mão-de-Obra e Geração de Emprego e Renda". Desde a criação do Programa GESTÃo PÚBLICA E CIDADANIA em 1996, essas foram as áreas que mais apresentaram iniciativas.

Isso pode ser explicado pelas próprias competências dos governos subnacionais e pelos processos de descentralização e municipalização em andamento no país, principalmente em áreas como educação e saúde, por exemplo. Além disso o ECA - Estatuto da Criança e Adolescente - determina que estas instâncias governamentais formulem e implantem políticas públicas voltadas a essa população. Outro estímulo à maior atuação dos governos locais na área social se deu com a criação de diversos Conselhos Estaduais e Municipais como de Educação, Saúde, Criança e Adolescente, Assistência Social, Emprego etc.

Em relação à área de "Criança e Adolescente", houve um aumento substancial de inscrições do ano de 1997 para 1998. Em 1999 o percentual das inscrições nesta área se manteve (15,5\% em 1998 e 15,9\% em 1999). A área de "Educação" apresentou, nestes quatro anos, o maior número de inscrições. Nos anos de 1996 e 1997 era esta a área que continha a maioria das iniciativas, representando $15,6 \%$ e $20,2 \%$ do total de inscrições, respectivamente. Em 1998, cai a participação relativa desta área, mas ela ainda se mantém em patamar significativo (11,7\% em 1998 e 13,7\% em 1999) (vide tabela 8). 
UMA BREVE ANÁLISE DO CICLO DE PREMIAÇÃO 1999

TABELA 8 - DISTRIBUIÇÃO DOS PROGRAMAS, PROJETOS E ATIVIDADES INSCRITOS, POR ÁREAS DE AC̣ÃO GOVERNAMENTAL - CICLOS DE PREMIAÇÃO 1996, 1997, 1998 E 1999

\begin{tabular}{|c|c|c|c|c|c|c|c|c|c|c|}
\hline \multirow{2}{*}{$\begin{array}{l}\text { área de ação } \\
\text { governamental }\end{array}$} & \multicolumn{2}{|c|}{ total } & \multicolumn{2}{|c|}{1996} & \multicolumn{2}{|c|}{1997} & \multicolumn{2}{|c|}{1998} & \multicolumn{2}{|c|}{1999} \\
\hline & n.a. & $(\%)$ & n.a. & $(\%)$ & n.a. & $(\%)$ & n.a. & $(\%)$ & n.a. & $(\%)$ \\
\hline total & 2.445 & 100,0 & 629 & 100,0 & 297 & 100,0 & 631 & 100,0 & 888 & 100,0 \\
\hline educação & 354 & 14,5 & 98 & 15,6 & 60 & 20,2 & 74 & 11,7 & 122 & 13,7 \\
\hline criança e adolescente & 316 & 12,9 & 50 & 8,0 & 27 & 9,1 & 98 & 15,5 & 141 & 15,9 \\
\hline saúde & 315 & 12,9 & 81 & 12,9 & 45 & 15,2 & 75 & 11,9 & 114 & 12,8 \\
\hline assistência social & 120 & 4,9 & 18 & 2,9 & 6 & 2,0 & 26 & 4,1 & 70 & 7,9 \\
\hline $\begin{array}{l}\text { cultura, patrimônio histórico } \\
\text { e artístico }\end{array}$ & 110 & 4,5 & 38 & 6,0 & 17 & 5,7 & 23 & 3,7 & 32 & 3,6 \\
\hline gestão e planejamento & 108 & 4,4 & 38 & 6,0 & 11 & 3,7 & 23 & 3,7 & 36 & 4,0 \\
\hline $\begin{array}{l}\text { formação de mão-de-obra, } \\
\text { geração de emprego e renda }\end{array}$ & 105 & 4,3 & 16 & 2,5 & 12 & 4,0 & 30 & 4,8 & 47 & 5,3 \\
\hline idoso & 64 & 2,6 & 10 & 1,6 & 6 & 2,0 & 16 & 2,5 & 32 & 3,6 \\
\hline lazer e esportes & 47 & 1,9 & -- & -- & 3 & 1,0 & 16 & 2,5 & 28 & 3,2 \\
\hline minorias & 42 & 1,7 & 10 & 1,6 & 1 & 0,3 & 10 & 1,6 & 21 & 2,4 \\
\hline demais áreas & 864 & 35,3 & 270 & 42,9 & 109 & 36,7 & 240 & 38,0 & 245 & 27,6 \\
\hline
\end{tabular}

As inscrições na área de "Saúde" mantiveram-se constantes, se comparadas as participações em relação ao total de inscrições em cada ano. As áreas de "Assistência Social" e "Formação de Mão-de-Obra e Geração de Emprego e Renda" mostraram um aumento de inscrições de 1998 para 1999. Em relação à área de "Assistência Social", o total de inscrições passou de 26, em 1998, para 70, em 1999. As inscrições na área de "Formação de Mão-de-Obra e Geração de Emprego e Renda" aumentaram de 30 para 47 entre 1998 e 1999.

TABELA 9 - PROGRAMAS, PROJETOS E ATIVIDADES INSCRITOS, POR ÁREA DE AÇÃO GOVERNAMENTAL SEGUNDO A REGIÃO GEOGRÁFICA CICLOS DE PREMIAÇÃO 1996، 1997, 1998 E 1999

\begin{tabular}{|c|c|c|c|c|c|c|c|c|c|c|c|c|}
\hline \multirow{2}{*}{$\begin{array}{l}\text { área de ação } \\
\text { governamental }\end{array}$} & \multicolumn{2}{|c|}{ total } & \multicolumn{2}{|c|}{ Norte } & \multicolumn{2}{|c|}{ Nordeste } & \multicolumn{2}{|c|}{ Sudeste } & \multicolumn{2}{|c|}{ Sul } & \multicolumn{2}{|c|}{ Centro-Oeste } \\
\hline & n.a. & (\%) & n.a. & $(\%)$ & n.a. & $(\%)$ & n.a. & $(\%)$ & n.a. & $(\%)$ & n.a. & $(\%)$ \\
\hline Total & 888 & 100,0 & 38 & 100,0 & 116 & 100,0 & 453 & 100,0 & 263 & 100,0 & 18 & 100,0 \\
\hline criança e adolescente & 141 & 15,9 & 4 & 10,5 & 17 & 14,7 & 83 & 18,3 & 30 & 11,4 & 7 & 38,9 \\
\hline educação & 122 & 13,7 & 3 & 7,9 & 15 & 12,9 & 53 & 11.7 & 50 & 19.0 & 1 & 5,6 . \\
\hline saúde & 114 & 12,8 & 2 & 5,3 & 13 & 11,2 & 58 & 12,8 & 40 & 15,2 & 1 & $5,6^{\circ}$ \\
\hline assistência social & 70 & 7,9 & 2 & 5,3 & 13 & 11,2 & 28 & 6,2 & 24 & 9,1 & 3 & 16,7 \\
\hline $\begin{array}{l}\text { formação de mão-de-obra, } \\
\text { geração de emprego e renda }\end{array}$ & 47 & 5.3 & 2 & 5,3 & 9 & 7,8 & 22 & 4,8 & 14 & 5,3 & -- & -- \\
\hline gestão e planejamento & 36 & 4,1 & 2 & 5,3 & 5 & 4,3 & 16 & 3,5 & 12 & 4,6 & 1 & 5,6 \\
\hline $\begin{array}{l}\text { cultura, patrimônio } \\
\text { histórico e artístico }\end{array}$ & 32 & 3,6 & 1 & 2,6 & 5 & 4,3 & 12 & 2,7 & 14 & 5,3 & -- & - \\
\hline lazer e esportes & 32 & 3,6 & 2 & 5,3 & 5 & 4,3 & 19 & 4,2 & 5 & 1,9 & 1 & 5,6 \\
\hline idoso & 28 & 3,2 & -- & -- & 4 & 3,4 & 14 & 3,1 & 9 & 3,4 & 1 & 5,6 \\
\hline minorias & 21 & 2,4 & 6 & 15,8 & -- & -. & 12 & 2,7 & 2 & 0,8 & 1 & 5,6 \\
\hline demais áreas & 245 & 27,6 & 14 & 36,8 & 30 & 25,9 & 136 & 30,0 & 63 & 24,0 & 2 & 11.1 \\
\hline
\end{tabular}


As áreas de "Criança e Adolescente" e "Assistência Social" foram as de maior destaque na região Centro-Oeste no Ciclo de Premiação 1999. Já a região Sul contou com a maior participação percentual de inscrições na área de "Educação" e "Saúde". A região Nordeste destacou-se na área de "Formação de Mão-de-Obra e Geração de Emprego e Renda" e a região Norte, na área de "Minorias", explicado pelo grande número de programas de organizações próprias de povos indígenas (vide tabela 9).

Com os dados apresentados, buscou-se fazer um levantamento geral das características relativas ao Ciclo de Premiação 1999, o quarto do Programa GESTÃo PÚBLICA E CIDADANIA. Cabe alertar que as considerações aqui desenvolvidas necessitam, para adquirir cunho analítico mais rigoroso, de pesquisa mais aprofundada. Não foi esta a intenção do esforço aqui empreendido, mas antes apenas apresentar alguns dados que permitam uma primeira aproximação do conjunto de iniciativas recebidas neste ano, traçandose algumas comparações com os três Ciclos anteriores.

\section{Bibliografia consultada}

BELTRÃO, Ricardo Ernesto Vasquez; FARAH, Marta Ferreira Santos \& LACZYNSKI, Patrícia. "Um primeiro olhar sobre o Ciclo de Premiação 1998". In: BELTRÃO, Ricardo Ernesto Vasquez; MAURO, Carlos Eduardo Evangelisti \& LACZYNSKI, Patrícia (org.). Cadernos Gestão Pública e Cidadania (A administração pública brasileira inovando a forma de governar: apresentação dos 631 programas inscritos no Ciclo de Premiação 1998). São Paulo: EAESP-FGV, v.12, 1998 , p.7-19.

FARAH, Marta Ferreira Santos. "Que está mudando na gestão pública brasileira? Tendência sugeridas pelos programas inscritos". In: BELTRÃO, Ricardo Ernesto Vasquez (org.). Cadernos Gestão Pública e Cidadania (A administração pública brasileira inovando a forma de governar Ciclo de Premiação 1996). São Paulo: EAESP-FGV, v.1, 1996, p.7-16. 
UMA BREVE ANÁLISE DO CICLO DE PREMIAC̣̃̃o 1999

FARAH, Marta Ferreira Santos \& BELTRÃO, Ricardo Ernesto Vasquez. "Perfil dos programas inscritos em 1997". In: BELTRÃO, Ricardo Ernesto Vasquez (org.). Cadernos Gestão Pública e Cidadania (A administração pública brasileira inovando a forma de governar: apresentação dos 297 programas inscritos no Ciclo de Premiação 1997). São Paulo: EAESP-FGV, v.8, 1996, p.7-14.

FUndAC̣Ão INSTITUTO BRASILEIRO DE GEOgRAFIA E ESTAtísticA - IBgE. Contagem Populacional - 1996. Rio de Janeiro, IBGE, 1996.

SPINK, Peter; CLEMENTE, Roberta \& KEPPKE, Rosane. Governo local: o mito e as novas práticas de governança. Revista de Administração. São Paulo, v.34, n.1, jan./mar.1999, p.61-9. 